\title{
O-SPIN: an Opportunistic Data Dissemination Protocol for Folk-enabled Information System in Least Developed Countries
}

\author{
R. Petrolo ${ }^{1}$, T. Delot ${ }^{1,2}$, N. Mitton ${ }^{1}$, A. Molinaro $^{3}$, C. Campolo ${ }^{3}$ \\ ${ }^{1}$ Inria, France ${ }^{2}$ LAMIH, University of Valenciennes, France \\ ${ }^{3}$ DIIES, Universitá Mediterranea di Reggio Calabria, Italy
}

\begin{abstract}
Without universal access to the Internet, Least Developed Countries are left by the wayside of the digital revolution. Research is underway to overstep the barrier to the development of information technology services in these areas. In this context, the Folk-IS (Folk-enabled Information System) is a new fully decentralized and participatory approach, in which, each individual can transparently perform data management and networking tasks through highly secure, portable, and lowcost storage and computing personal devices, as physically moving, so that global services can finally be delivered by crowd. In this paper, we propose Opportunistic SPIN (O-SPIN), an information dissemination protocol that augments the well-known data-centric energy-aware SPIN (Sensor Protocols for Information via Negotiation) protocol to enable networking facilities for Folk-nodes, by exploiting opportunistic contacts among users. Performance of the proposed solution has been evaluated through simulations carried out in the OMNeT ++ framework under different settings. Achieved results demonstrate its effectiveness and efficiency in the information dissemination process.
\end{abstract}

\section{Introduction}

Today, citizens in developed countries receive payslips, banking statements, medical records, and other personal data through the Internet. The advantages are numerous, to name a few, e-services are faster, more convenient, and can be used to save time, strengthen the markets, improve the quality of life, etc. Unfortunately, this is not yet possible in Least Developed Countries (LDCs), where the capillary diffusion of Information and Communication Technology (ICT) is still hindered by several social, technical, and economical barriers.

According to Non-Governmental Organizations (NGOs), four main requirements must be met to build a practical technical solution in LDCs: privacy protection, immediate personal benefit, self-sufficiency, and very low deployment

\footnotetext{
* This work has been partially supported by the PalmaRES Project, funded by MIUR - Cooperlink initiative and by CPER NPdC/FEDER CIA, PREDNET LIRIMA and the FP7 VITAL.
} 
cost. To comprehensively address the mentioned issues, a paradigm called Folkenabled Information System (Folk-IS) has been preliminarily introduced in [1]. Folk-IS promotes the idea of an infrastructure-less and participatory platform, where each individual implements a small subset of the complete information system, thanks to emerging highly secure, portable, and low-cost storage as well as computing personal devices, called Smart Tokens. Within Folk-IS, people transparently and opportunistically performs data management and networking tasks as they physically move, thus enabling human networking. Human networks are a special kind of opportunistic networks, in which people exchange information when they meet. Opportunistic networking is, indeed, claimed to be the only affordable way to help bridging the digital divide by providing intermittent Internet connectivity to rural and developing areas $[2,3]$. However, the design of simple and efficient opportunistic routing and data dissemination strategies is, generally, a complicated task due to the lack of knowledge about the topological evolution of the network. Routes are built dynamically while messages are en route between the sender and the destination(s), and any node can opportunistically be used as a next-hop, provided it is likely to bring the message closer to the final destination.

In order to contribute to this issue, in this paper, an opportunistic data dissemination protocol is proposed to enable the Folk-IS paradigm. The proposed Opportunistic SPIN (O-SPIN) takes advantages of both data-centric and opportunistic routing philosophies. O-SPIN borrows the main features of SPIN (Sensor Protocols for Information via Negotiation), a family of data-centric routing protocols used to efficiently disseminate information in Wireless Sensor Networks (WSNs) $[4,5]$. The rationale behind our design choice is as follows:

- Being deployed for WSNs, SPIN is designed to keep the energy consumption as low as possible. This is an attractive feature for Folk-IS nodes equipped with resource-constrained battery-powered Smart Tokens.

- Targeting the delivery of sensor data, SPIN is optimized for the single exchange of small-sized packets. This is the typical data exchange mainly targeted in FolkIS scenarios, like public utility data (e.g., drought warnings, humanitarian aids advertisements), e-administration data (e.g., recording of births and deaths).

To fit the requirements of Folk-IS scenarios, experiencing intermittent connectivity due to a variety of reasons, O-SPIN is enhanced to exploit the opportunistic contacts among personal devices. To serve this purpose, periodical advertisement that supports the dissemination process and neighbourhood information exchange are additionally foreseen.

The remainder of the paper is organized as follows. Section II presents the Folk-IS paradigm and the Smart Token, the requirements of the data dissemination protocol and use cases. Section III describes the proposed O-SPIN protocol. Performance comparison between O-SPIN and a variant of SPIN are reported in Section IV. Section V concludes the paper and provides hints for future work. 


\section{Preliminaries: The Folk-IS paradigm and architecture}

Alongside good governance, technology is considered among the greatest enablers for improving quality of life. However, the majority of its benefits have been concentrated in industrialized nations and are thus limited to a world's population fraction. E-services could be considered superfluous luxury for LDCs, where population makes less than $\$ 2,000$ per year [6]. On the contrary, several reports [7-9] make evident that ICT are called to play a catalytic role in these countries. ICT can help to achieve universal primary education, promote gender equality and empower woman, reduce child mortality, combat diseases or ensure environmental sustainability. However, making e-services practical and affordable is still challenging because of lack of infrastructure, high initial investment, difficulty to maintain the system operational, reluctance to use the system due to security concern, etc.

The Folk-IS paradigm aims to address four main requirements [1]:

- Ethic 83 security: the lack of a strict legal framework regulating people's privacy leads to recognize an LDC as an especially hostile context. Privacy abuses, data corruption and denial of service attacks, often driven by financial or political interests or ethnic disputes, are usually not deterred by sufficiently coercive laws. Thus, privacy protection and security are considered by NGOs as two strong prerequisites before deploying any solution in the field;

- User's benefit: providing a global interest for the community is not a sufficient incentive. The solution must provide a direct benefit to each user to be effectively used in the field;

- Self-sufficiency: relying on the expected improvement of the technical infrastructure or on upcoming governmental programs or laws implies a major risk of failure since not perennial;

- Sustainability: the deployment cost of the solution must be very low (a few dollars per user) and proportional to the life's cost of those areas, without huge initial investment. The maintenance cost must also be minimal and should ideally generate a source of revenues for new local jobs linked to the solution.

Folk-IS builds upon the emergence of highly secure, portable and low-cost storage and computing devices called Smart Tokens (Figure 1(a)). These devices combine the tamper-resistance of a secure micro-controller with high storage capacity external NAND flash chips and short-range communication capabilities. The Smart Token considered in this paper is very similar to a smart token product, provided by Gemalto, used by Inria in a field experiment [10]. This kind of smart token is available for a few dollars, in a SIM card form factor (plugged in a USB key casing), and simply needs to be extended with a fingerprint reader, in a similar way to traditional secure USB keys. In our context, the Smart Token is more complex, since it embeds a battery (and the required means to charge it) and powers a wireless communication element (Bluetooth, led-light communications, etc.). It is also equipped with $\mathrm{I} / \mathrm{O}$ resources to allow basic users interacts autonomously. 


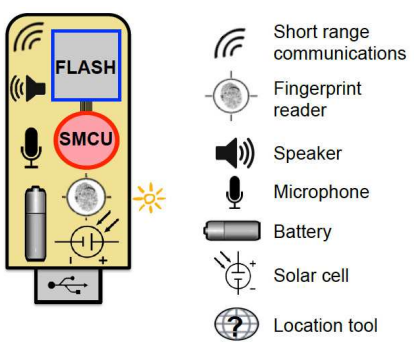

(a) Smart Token.

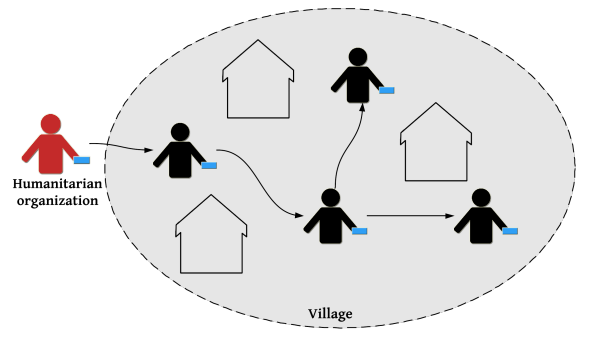

(b) Mode of operation.

Fig. 1: Folk-IS paradigm.

Folk-IS is characterized by the following features:

- Infrastructure-less environment: nodes cooperatively establish the network independently of any fixed base station infrastructure.

- Distributed communication: each individual implements a small subset of the complete information system; at the same time, a node can act as a user of the system and also as a human network node. There is no central coordinator for the communications among humans that are typically ad-hoc.

- Mobility: nodes move inside the area more or less randomly so, the topology is highly dynamic. Moreover, Smart Tokens carried by pedestrians are not equipped with GPS-like receivers due to cost/energy requirements. Hence, human communications should take advantage from recurrent mobility patterns (e.g., a bus) to improve message delivery performance.

- Resource-constrained devices: Smart Tokens are devices with several limitations: (i) Low-cost: the deployment cost must be very low (a few dollars each) in order to be suitable for those countries; (ii) Low-energy: the devices must be able to power the wireless communication component and the other functionalities while consuming less battery as possible; (iii) Hardware constraints: the devices have small capacity, like a tiny RAM (about $64 \mathrm{kB}$ ), a small micro-controller and a small internal stable storage. Thus, it is fundamental to well manage the information stored in the device, removing the old one in order to free up space.

Many applications can benefit of the Folk-IS paradigm, provided that they are compliant with high-latency asynchronous data exchanges. Let us imagine a humanitarian organization in a LDC that wants to inform the population about the date for medical checks and vaccinations. As shown in Fig. 1(b), it transmits this information through the ad-hoc Folk-enabled Network (Folk-Net). Every Folk-node associated to an individual acts as a human network node and forwards the message to its neighbours.

\subsection{Data dissemination in Folk-Net}

To match the peculiarities of Folk-IS scenarios, routing should be:

- data-centric: all the interest is in the data, not in the location/identity of the node. This is because it is not possible to exactly track position of individuals since Smart Tokens are not equipped with a GPS. 
- energy-efficient: personal devices are battery-powered and might be not rechargeable often because of the lack of charging points in LDCs.

- opportunistic: Communications can follow a carry-and-forward approach. Given the lack of infrastructure and the highly dynamic nature of Folk-Net, people will transparently perform networking tasks as they physically move, by exploiting the short-lived contacts with encountered users.

Several protocols in the literature may match the aforementioned requirements. Various data dissemination schemes have been proposed for delay-tolerant and opportunistic networks and energy saving protocols for resource-constrained WSNs [11], [12]. Among the two most representative energy-efficient data-centric approaches, i.e., Directed Diffusion [13] and SPIN [4], the latter, proposed by Heinzelman et al. in [4] and [5], is a family of adaptive protocols that disseminate the sensed information to every interested node in the network by using meta-data advertisement and negotiation to favour aggregation, redundancy elimination and energy saving [11]. The conceived data dissemination protocol, O-SPIN, borrows its main tenets from the SPIN protocol proposed for WSNs. By doing so, it provides data-centric and energy-efficient communications, and it is augmented to leverage opportunistic contacts among users to disseminate data.

\section{The proposed Opportunistic SPIN}

We now describe the designed O-SPIN protocol, by emphasizing aspects in common and differences with the SPIN-2 protocol in [4] (hereafter shortened as SPIN).

\section{$3.1 \quad$ O-SPIN in a nutshell}

Opportunistic data delivery in O-SPIN occurs in the following three steps: Data Advertisement, Data Request, and Data Delivery, as shown in Figure 2(a). The organization in three steps is shared with traditional SPIN, but the message format and the behavior details of each phase are different. Each O-SPIN node keeps the following data structures: the Neighbours Table, storing information about encountered neighbours, and the Data Table, storing retrieved data and related meta-data that describes the data content.

Data Advertisement. In traditional SPIN, a sensor node advertises new data only when they are sensed by the node itself or received by neighbouring nodes. So the broadcasting of advertisement (ADV) messages is asynchronous. The first main difference between SPIN and O-SPIN consists in the introduction of periodical advertisements. Since human networking context is mobile and sparse, when a node transmits the ADV, it may not find any neighbour. The introduction of periodical advertisements (every $A D V$ Message Interval) provides a more efficient information dissemination, as demonstrated in Section 4, on the other hand, it implies higher energy consumption. 
Data Request. When a node receives an ADV Message, it checks whether it already holds the corresponding Data; otherwise, it sends a Request (REQ) Message to ask for it. REQ is sent if the following conditions hold: the destination of the packet is the node itself, $O R$ the destination of the packet is the entire community, $O R$ somehow the node knows how to reach the destination, $A N D$ the residual energy in the node allows it to complete the operation.

Data Delivery. When the advertising node receives a REQ Message, it sends back the DATA Message. Data can then be stored by the receiving node and further forwarded hop-by-hop to the destination.

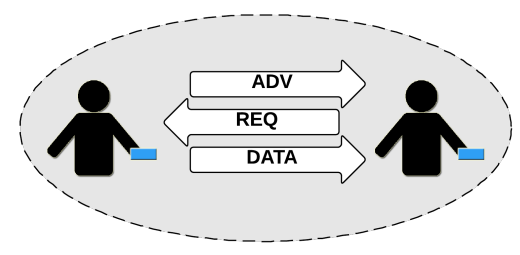

(a) Basic phase.

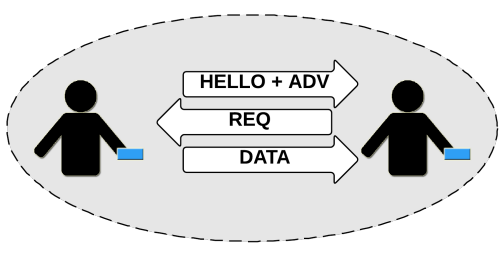

(b) Ehnanced phase.

Fig. 2: O-SPIN basic (a) and enhanced (b) phases.

\subsection{Neighbour Discovery}

Contrarily to a static typical WSN scenario where neighbourhood information is easy to get, in the scenario under analysis, the neighbourhood of each Folk-Node can change more or less rapidly. Especially if the network is sparse, the risk is that source nodes and potential forwarders (or destination) hardly meet or even if they meet they do not realize it (e.g., because they miss the ADV message). O-SPIN makes nodes broadcast HELLO Messages every Hello Message Interval regardless of the availability of Data to help neighbour discovery and enjoy opportunistic contacts among users. When a node receives a HELLO Message, it updates its Neighbours Table. If a node has new data to transmit, it piggybacks it in its HELLO Message and decreases the Hello Message Interval to the ADV Message Interval . These new HELLO messages also contain the ADV for the Data (Fig. 2(b)). If a node does not receive a REQ for the Data advertised in the HELLO Message, for a time equal to four times the ADV Message Interval, it decreases the Hello Message Interval to the ADV Message Interval. All in all, O-SPIN changes the Advertisement step of traditional SPIN by (i) making the advertisement periodic, (ii) adding new messages (HELLO), and (iii) updating the Neighbours Table at every interaction between nodes.

\subsection{Energy Conservation}

O-SPIN implements a simple energy-conservation heuristic similar to SPIN-2 [5]. When nodes are energy plentiful, they work normally by following the three-way handshake protocol (ADV-REQ-DATA), as described in the previous sections. 
Otherwise, if a node observes that its energy reaches a low threshold, it reacts by reducing its participation in the protocol. Specifically, a node will only participate in a protocol stage if it can complete all the successive stages without going below the low-energy threshold. This conservative approach implies that, if a node receives some new data, it only initiates the ADV phase if it has enough energy to participate in the full three-stage protocol with all its neighbours. Similarly, if a node receives an ADV, it does not send out a REQ unless it has enough energy to both send a REQ and receive Data. This approach does not prevent a node from spending energy in receiving ADV or REQ messages even when it is below its low-energy threshold. It does, however, prevent the node from ever handling a DATA message when it runs out of energy.

\subsection{O-SPIN Packets and Data Structures}

O-SPIN uses 4 types of messages (Fig. 3): HELLO, ADV, REQ, and DATA.

HELLO Packet. A HELLO packet (Fig. 3a) contains the user identifier (USER ID), unique in the entire network, and the home town (Home Town). When a node $u$ receives a HELLO Message from $v$, it stores information about $v$ in its Neighbours Table and, eventually, updates the number of times (Number of MeEtings) and the last time (LAST MeEting) $v$ has been met, which allow removal of expired entries and additional criteria for the next-hop selection. More details are given in Section 3.5.

\begin{tabular}{|c|c|c|c|c|c|c|c|c|}
\hline \multirow[t]{2}{*}{ Octets: } & 7 & 7 & \multirow{2}{*}{\multicolumn{2}{|c|}{ (a) }} & \multirow[b]{3}{*}{7} & \multirow[b]{3}{*}{1} & \multirow[b]{3}{*}{2} & \multirow[b]{3}{*}{1} \\
\hline & User ID & $\begin{array}{l}\text { Home } \\
\text { Town }\end{array}$ & & & & & & \\
\hline \multirow[t]{2}{*}{ Octets: } & 7 & 7 & 7 & 7 & & & & \\
\hline & User ID & $\begin{array}{l}\text { Creator } \\
\text { ID }\end{array}$ & $\begin{array}{c}\text { Creator } \\
\text { Home } \\
\text { Town }\end{array}$ & $\begin{array}{l}\text { Destination } \\
\text { ID }\end{array}$ & $\begin{array}{c}\text { Destination } \\
\text { Town }\end{array}$ & $\begin{array}{c}\text { Data } \\
\text { Packet } \\
\text { ID }\end{array}$ & $\begin{array}{c}\text { Data } \\
\text { Description }\end{array}$ & $\begin{array}{c}\text { Data } \\
\text { Lifetime }\end{array}$ \\
\hline \multirow[t]{2}{*}{ Octets: } & 7 & 7 & 1 & & & & & \\
\hline & User ID & $\begin{array}{l}\text { Creator } \\
\text { ID }\end{array}$ & $\begin{array}{l}\text { Data } \\
\text { Packet } \\
\text { ID }\end{array}$ & (c) & & & & \\
\hline \multirow[t]{2}{*}{ Octets: } & 7 & 7 & 7 & 7 & 7 & 1 & $0-66$ & \\
\hline & User ID & $\begin{array}{l}\text { Creator } \\
\text { ID }\end{array}$ & $\begin{array}{c}\text { Creator } \\
\text { Home } \\
\text { Town }\end{array}$ & $\begin{array}{l}\text { Destination } \\
\text { ID }\end{array}$ & $\begin{array}{l}\text { Destination } \\
\text { Town }\end{array}$ & $\begin{array}{l}\text { Data } \\
\text { Packet } \\
\text { ID }\end{array}$ & Data & (d) \\
\hline
\end{tabular}

Fig. 3: O-SPIN messages: HELLO (a), HELLO+ADV (b), REQ (c), DATA (d).

$H E L L O+A D V$ Packet. When a node $u$ gets new data from a node $v$, it adds the Data in the HELLO message issuing a HELLO+ADV message (Fig. 3b), which actually is an ADV sent periodically. The fields of this HELLO+ADV packet are:

- User ID, the identifier of $u$;

- Creator ID, the identifier of $v$;

- Creator Home Town, the town of residence of $v$; 
- Destination ID, the identifier of the ultimate destination node; it can be a broadcast address if the message is to be spread among all the population;

- Destination Town, the destination town of the DATA packet, if specified;

- Data Packet ID, the identifier of the Data Packet (unique);

- Data Description, is an optional meta-data description of the data content;

- Data Lifetime, it represents the lifetime of a data packets, once obsolete, these packets should not be considered any more.

REQ Packet. When a node receives a HELLO+ADV message, it first updates its Neighbours Table and then checks whether it already has the Data, otherwise, it sends a REQ message (Figure 3c) to specify the Data it is interested into.

DATA Packet and Data Table. When a node $u$ receives a REQ message, first it updates its Neighbours Table, and then sends the DATA message (Fig. 3d). Once the DATA message is received, $u$ stores the following information in its Data Table: DATA Packet ID; Creator ID; Creator Home Town; Destination ID (representing the identifier of the destination user); Destination Home Town; Data (representing the information to be disseminated); RECEIVED Times (date of Data reception). If the destination of the received message is $u$ itself, it consumes the Data; otherwise, it starts to send HELLO+ADV Messages to advertise the new Data.

\subsection{Next-Hop Selection Criteria}

Some fields of the O-SPIN packets like Destination ID, Destination Town, Home Town, Number of Meetings and Last Meeting are fundamental in the routing strategies. Some possible usage examples are identified in this section; the different routing strategies depend on the application type. For example, public utility applications require data to be broadcast to the largest population in the shortest time (e.g., vaccination advertisements); e-administration applications usually target a specific village (e.g., birth recording); and so on. When a node receives a HELLO+ADV message, it may send a REQ message (and hence candidate itself as a possible forwarder) if one of these conditions is met, for example: the destination of the packet is its direct neighbour; the destination of the packet is its most-frequently met neighbour; the destination of the packet is a neighbour that has been very recently met; the destination town of the packet is its residence town; the destination town of the packet is the residence town of one of its neighbours.

In the simulation study presented in the next section we have implemented just few of the possible routing policies by focusing on the broadcasting of a message that is useful for the entire community.

\section{Performance Evaluation}

\subsection{Simulation settings}

To evaluate the performance of the proposed O-SPIN protocol we used OMNeT $++{ }^{1}$ and the simulation framework Castalia ${ }^{2}$. We consider a village as an area of $500 \times 500 \mathrm{~m}^{2}$

\footnotetext{
${ }^{1}$ http://www. omnetpp.org

${ }^{2}$ http://castalia.research.nicta.com.au/index.php/en/
} 
in which $N$ pedestrian nodes, equipped with a Smart Token, move according to the Random Waypoint Model, with an average speed of $1 \mathrm{~m} / \mathrm{s}$. Nodes are generated inside the village by using a uniform distribution. Simulations have been conducted under two different population densities: Low density, with $N=50$ nodes (200 persons per $\mathrm{km}^{2}$ ); High density, with $N=100$ nodes (400 persons per $\mathrm{km}^{2}$ ).

For each simulated scenario, we varied the $A D V$ Message Interval from 5 to $40 \mathrm{~s}$; Hello Message Interval from 10 to $80 \mathrm{~s}$. The initial energy budget of a Smart Token is set to 18720 Joules, which is the typical energy of two AA batteries. Only the radio component consumes energy for transmission, reception and carrier sensing. The Smart Tokens's RF Transmission Power is a varying parameter in our simulations; it is set to $0 \mathrm{dBm}$ and $-10 \mathrm{dBm}$, corresponding to a power consumption of $57.42 \mathrm{~mW}$ and $36.3 \mathrm{~mW}$, respectively. Table 1a reports the power transmission matrix with information on the power spent by each device in each status. In this paper the Duty Cycle, the fraction of time that the node listens to the channel and does not sleep, is set to 0.005, so nodes sleep for $1990 \mathrm{~ms}$. The nodes are not aligned in their sleeping schedules, so a train of beacons is sent before each data transmission to wake up potential receivers. Table 1b summarizes further Smart Token parameters, based on the Texas Instruments CC2400 datasheet.

Table 1: Simulator parameters.

\begin{tabular}{|c|c|c|c|c|c|}
\hline & & & & Parameter & Value \\
\hline & & & & Data Rate & $250 \mathrm{kbps}$ \\
\hline$\#$ & $\mathbf{R x}$ & $\mathbf{T x}$ & Sleep & Modulation & PSK \\
\hline $\mathbf{R x}$ & - & 62 & 62 & Bits per symbol & 4 \\
\hline $\mathbf{T x}$ & 62 & - & 62 & Bandwidth & $20 \mathrm{MHz}$ \\
\hline Sleep & 1,4 & 1,4 & - & Noise Bandwidth & $194 \mathrm{MHz}$ \\
\hline \multirow{2}{*}{\multicolumn{4}{|c|}{ (a) Power transition matrix $(\mathrm{mW})$. }} & Noise Floor & $-100 \mathrm{dBm}$ \\
\hline & & & & Sensitivity & $-95 \mathrm{dBm}$ \\
\hline
\end{tabular}

In the scenario under study, a node in a village creates a data packet of public utility; the goal is to spread the information in the entire village and inform the highest number of persons before the Smart Tokens run out of energy. The following metrics have been computed to compare the performance of the two protocols, in terms of effectiveness and efficiency of the data dissemination process:

- Percentage of informed nodes (PIN): representing the number of reached nodes, computed like: $P I N=\left(\frac{\text { Numberof NodesThatReceivedData }}{N}\right) * 100$.

-Energy Consumption (ECons): representing the average energy consumption per node, computed as ECons $=\left(\frac{\sum_{i=0}^{N}\left(\text { Energy Consumed }_{i}\right)}{N}\right)$.

Simulation results are reported with the $99 \%$ confidence intervals.

\subsection{Simulation results}

A first analysis showed us that the original version of SPIN, traditionally designed for a static WSN scenario, performs very poorly, i.e., after 1 hour of simulation, only two nodes received the Data packet, regardless of the transmission power $(0$ or $-10 \mathrm{dBm})$. Therefore, for the sake of equity and fairness, we have introduced some simple improvements to SPIN that we call SPIN-Enhanced with our O-SPIN. In SPIN-Enhanced the 
Table 2: Main features of benchmarked protocols.

\begin{tabular}{cccc}
\hline Protocol & $\begin{array}{c}\text { Hello } \\
\text { Exchange }\end{array}$ & ADV Exchange & $\begin{array}{c}\text { Energy conservation } \\
\text { heuristic }\end{array}$ \\
\hline $\begin{array}{c}\text { SPIN- } \\
\text { Enhanced }\end{array}$ & N/A & periodical & supported \\
\hline O-SPIN & periodical & $\begin{array}{c}\text { periodical with } \\
\text { adaptive repetition } \\
\text { interval }\end{array}$ & supported \\
\hline
\end{tabular}

advertisement process is regularly repeated at a fixed interval once new Data is created or received, and the same energy conservation heuristic as SPIN is implemented. For the sake of clarity, the main features of the benchmarked solution are summarized in Table 2 .

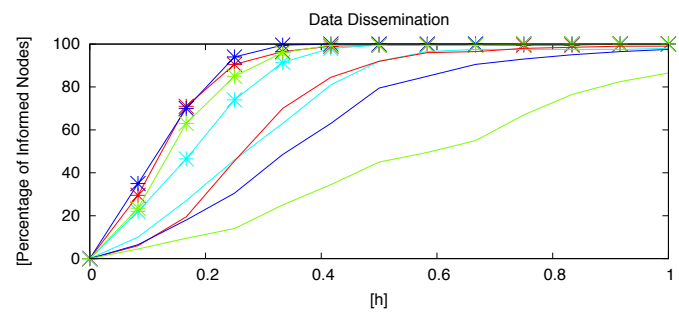

(a) Data Dissemination effectiveness.

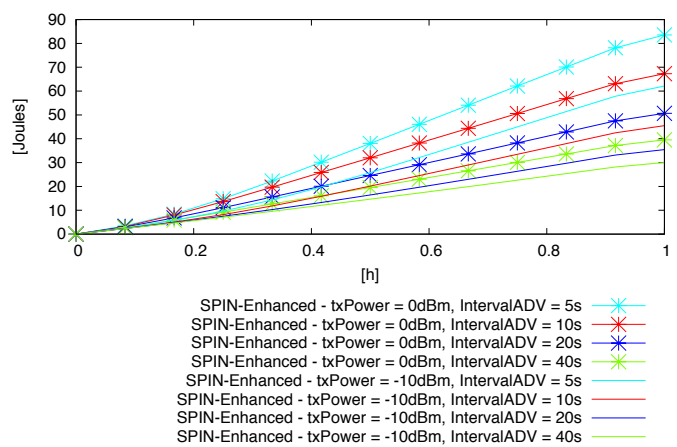

(b) Energy Consumption.

Fig. 4: SPIN-Enhanced performance (low density).

SPIN-Enhanced (low density population). As shown in Figure 4(a), SPIN-Enhanced with Transmission Power of $0 \mathrm{dBm}$ presents better performance than the case with Transmission Power of $-10 \mathrm{dBm}$. In the former case, all nodes inside the area receive the packet after about 30 minutes; this is because the transmission range of the Smart Token is larger. Moreover, it is possible to observe that the performance improves when the ADV Message Interval is shorter, except for the case with ADV Message Interval equal to $5 \mathrm{~s}$. Intuitively, when the interval is shorter the probability to exploit opportunistic contacts increases; however, if this interval is too short the number of 
transmitted ADV messages creates congestion on the channel. Finally, the effect of ADV Message Interval on data dissemination is more visible when the Transmission Power is $-10 \mathrm{dBm}$.

Figure 4(b) shows the relative Energy Consumption when changing Transmission Power and ADV Message Interval. When the Transmission Power is $0 \mathrm{dBm}$ the energy consumption is higher. Also, when ADVs are sent more frequently the energy consumption increases. Using SPIN-Enhanced we need to find a good trade-off between the parameters. So we use the ADV Message Interval equals to $10 \mathrm{~s}$ to achieve good performance when the Transmission Power is 0 and when it is $-10 \mathrm{dBm}$.

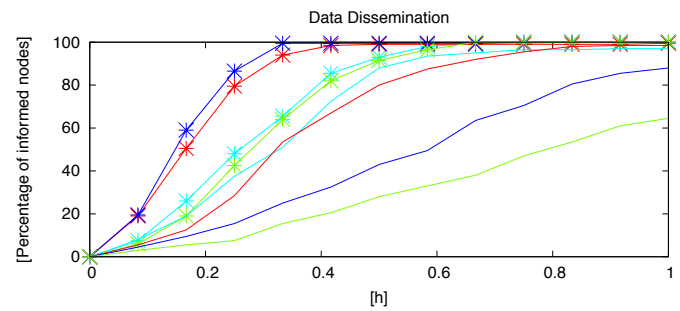

(a) Data Dissemination effectiveness.

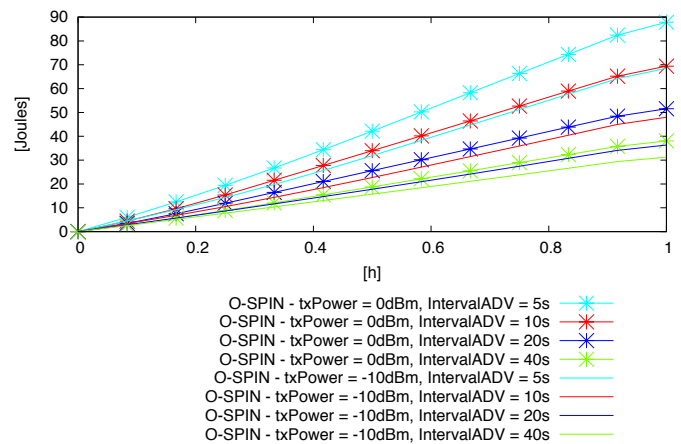

(b) Energy Consumption.

Fig. 5: O-SPIN performance (low density).

O-SPIN (low density population). Figure 5(a) shows the trend of Data Dissemination effectiveness when using our proposal. It is possible to observe also in this case that when the Transmission Power is set to $0 \mathrm{dBm}$ the performance is better. Also in this case, the performance decreases when the ADV Message Interval is equal to $5 \mathrm{~s}$. Figure 5(b) shows the Energy Consumption; again sending the ADV Messages with higher frequency corresponds to higher battery consumption.

SPIN-Enhanced vs. O-SPIN (low density population). We compare the performance of O-SPIN and SPIN-Enhanced under the simulation assumptions considered above, but excluding the case with ADV Message Interval of $5 \mathrm{~s}$ because it is a lowperforming case for both protocols. Both protocols are able to inform all the network nodes in one hour (Figure 6(a)). In most cases, O-SPIN is slightly slower to diffuse the information, but we can observe that when the ADV Message Interval is set to $10 \mathrm{~s}$, both 
protocols achieve similar performance. Figure 6(b) compares the Energy Consumption between the two routing protocols. O-SPIN spends less energy than SPIN-Enhanced. Figure 7 shows the network lifetime using Transmission Power to $0 \mathrm{dBm}$ and the Interval ADV to $10 \mathrm{~s}$. The assumption is that a single node creates a Data Packet each hour. The curves show that Smart Tokens running O-SPIN have 1 day more of autonomy compared with devices which run SPIN-Enhanced, which is a significant improvement since devices might be not recharged often.

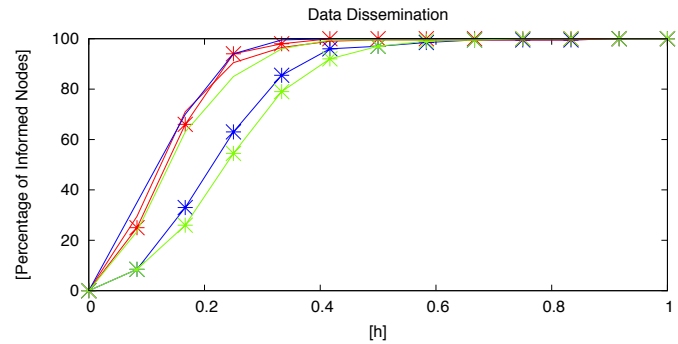

(a) Data Dissemination effectiveness.

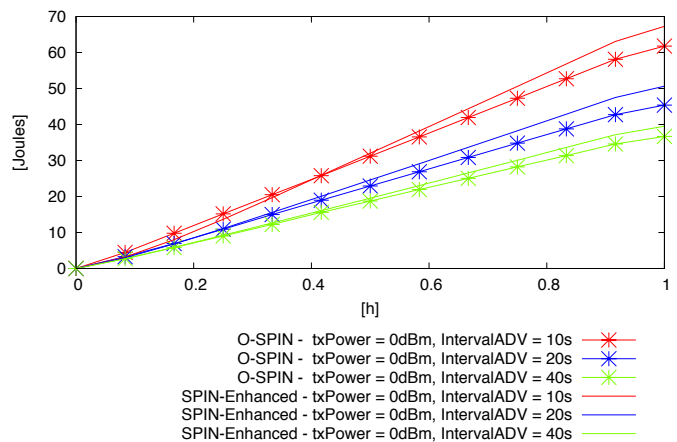

(b) Energy Consumption.

Fig. 6: O-SPIN vs. SPIN-Enhanced performance (low density).

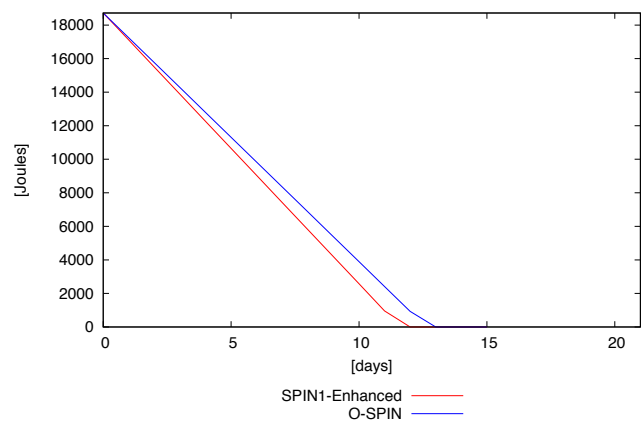

Fig. 7: O-SPIN vs. SPIN-Enhanced Network lifetime. 
SPIN-Enhanced vs. O-SPIN (high density population). In this section, we compare the performance of SPIN-Enhanced and O-SPIN protocols in the high density scenario. Figure 8(a) shows that in 30 min all nodes receive the Data packet. O-SPIN performance increases when the ADV Interval is smaller. Figure 8(b) shows the Energy Consumption; O-SPIN saves more energy than SPIN-Enhanced.

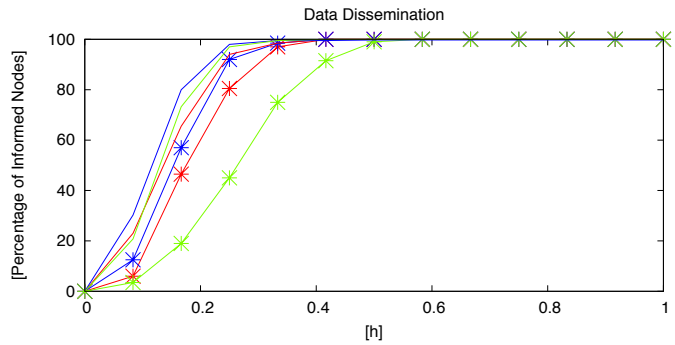

(a) Data Dissemination effectiveness.

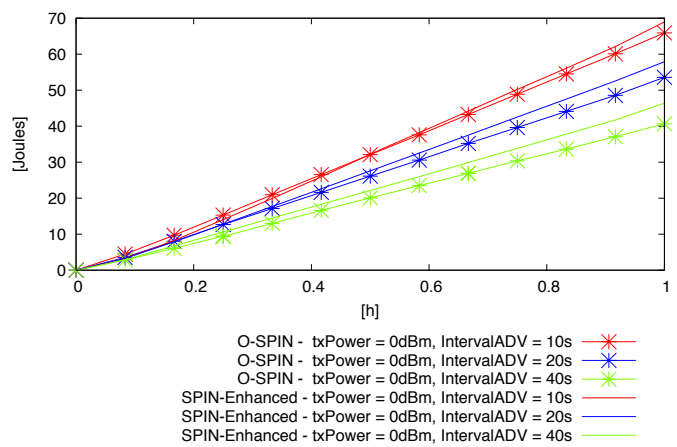

(b) Energy Consumption.

Fig. 8: O-SPIN vs. SPIN-Enhanced (high density).

\section{Conclusion}

In this paper a new routing technique to enable human networking in LDC was presented. Opportunistic contacts between people equipped with a personal device, empowered with communication and storing capabilities, allow information dissemination (e.g., data of public utility, like the presence of drinkable water, healthcare service, humanitarian aids, etc.) in an efficient and effective way. An important feature for these newly developed personal devices is the battery consumption. Indeed, it is fundamental to design communication and data exchange protocols that preserve the devices' battery. At the same time, it is also required that these devices are able to disseminate information to as many people as possible. We proposed a new routing protocol that enhances SPIN by exploiting the opportunistic contacts among personal devices.

We evaluated performance in different density scenarios, under different settings for transmission power and repetition intervals of messages. The results show that the proposed O-SPIN outperforms the straightforwardly enhanced version of SPIN, in terms 
of effectiveness and efficiency of the dissemination process with beneficial effects on the network lifetime. Transmission Power is the most important parameter that influences the performance metrics; indeed if it is high then both data dissemination and battery consumption are faster. Another important parameter is the ADV Message Interval, which indicates the periodicity of the ADV Messages. By increasing this interval, both data dissemination and battery consumption are slower.

In the future, we plan to study more sophisticated criteria for the next hop selection, and to introduce the adaptive setting of the ADV Message Interval based on traffic load, scenario characteristics, and node capability. We expect that the proposed Folk-IS vision may help to bridge the digital divide while pointing out research directions and solutions for efficient and effective information dissemination in hostile environments.

\section{References}

1. Anciaux, N., Bouganim, L., Delot, T., Ilarri, S., Kloul, L., Mitton, N., Pucheral, P.: Folk-IS: Opportunistic Data Services in Least Developed Countries. In: VLDB 2014 - 40th International Conference on Very Large Data Bases. (September 2014)

2. Pentland, A., Fletcher, R., Hasson, A.: DakNet: rethinking connectivity in developing nations. Computer 37(1) (January 2004) 78-83

3. Doria, A., Uden, M., Pandey, D.P.: Providing connectivity to the Saami nomadic community. In: Development by Design Conference. (2002)

4. Heinzelman, W.R., Kulik, J., Balakrishnan, H.: Adaptive protocols for information dissemination in wireless sensor networks. In: Proceedings of the 5th annual ACM/IEEE international conference on Mobile computing and networking - MobiCom '99, New York, New York, USA, ACM Press (August 1999) 174-185

5. Kulik, J., Heinzelman, W., Balakrishnan, H.: Negotiation-based protocols for disseminating information in wireless sensor networks. Wireless Networks $\mathbf{8}(2 / 3)$ (March 2002) 169-185

6. Brewer, E., Demmer, M., Du, B., Ho, M., Kam, M., Nedevschi, S., Pal, J., Patra, R., Surana, S., Fall, K.: The case for technology in developing regions. Computer 38(6) (May 2005) 25-38

7. Coceres, R., Belding, E., Parikh, T., Subramanian, L.: Information and Communication Technologies for Development [Guest editors' introduction]. IEEE Pervasive Computing 11(3) (March 2012) 12-14

8. ITU: The Role of ICT in Advancing Growth in Least Developed Countries - Trends, Challenges and Opportunities

9. Rossi, G., Murugesan, S., Godbole, N.: IT in Emerging Markets. IT Professional 14(4) (July 2012) 2-3

10. Allard, T., Anciaux, N., Bouganim, L., Pucheral, P., Thion, R.: Pervasive and Smart Technologies for Healthcare. IGI Global (March 2010)

11. Watteyne, T., Molinaro, A., Richichi, M.G., Dohler, M.: From MANET To IETF ROLL Standardization: A Paradigm Shift in WSN Routing Protocols. IEEE Communications Surveys \& Tutorials 13(4) (2011) 688-707

12. Akkaya, K., Younis, M.: A survey on routing protocols for wireless sensor networks. Ad hoc networks 3(3) (2005) 325-349

13. Intanagonwiwat, C., Govindan, R., Estrin, D.: Directed diffusion: a scalable and robust communication paradigm for sensor networks. In: Proceedings of the 6th annual international conference on Mobile computing and networking, ACM (2000) $56-67$ 